\title{
Nitrogen acceptor in $2 \mathrm{H}$-polytype synthetic $\mathrm{MoS}_{2}$ assessed by multifrequency electron spin resonance
}

Ben Schoenaers, Andre Stesmans, and Valery V. Afanas'ev

Citation: Journal of Vacuum Science \& Technology A 36, 05G503 (2018); doi: 10.1116/1.5034447

View online: https://doi.org/10.1116/1.5034447

View Table of Contents: http://avs.scitation.org/toc/jva/36/5

Published by the American Vacuum Society

\section{Articles you may be interested in}

Thermal recrystallization of short-range ordered $\mathrm{WS}_{2}$ films

Journal of Vacuum Science \& Technology A 36, 05 G501 (2018); 10.1116/1.5036654

Atmospheric pressure plasma jet: A facile method to modify the intimal surface of polymeric tubular conduits Journal of Vacuum Science \& Technology A 36, 04F404 (2018); 10.1116/1.5023259

Comparison of thermal and plasma-enhanced atomic layer deposition of niobium oxide thin films Journal of Vacuum Science \& Technology A 36, 041503 (2018); 10.1116/1.5034097

Graphene and related two-dimensional materials: Structure-property relationships for electronics and optoelectronics

Applied Physics Reviews 4, 021306 (2017); 10.1063/1.4983646

Review Article: Progress in fabrication of transition metal dichalcogenides heterostructure systems Journal of Vacuum Science \& Technology B, Nanotechnology and Microelectronics: Materials, Processing, Measurement, and Phenomena 35, 030803 (2017); 10.1116/1.4982736

P-type conduction in two-dimensional $\mathrm{MoS}_{2}$ via oxygen incorporation Applied Physics Letters 110, 193103 (2017); 10.1063/1.4983092

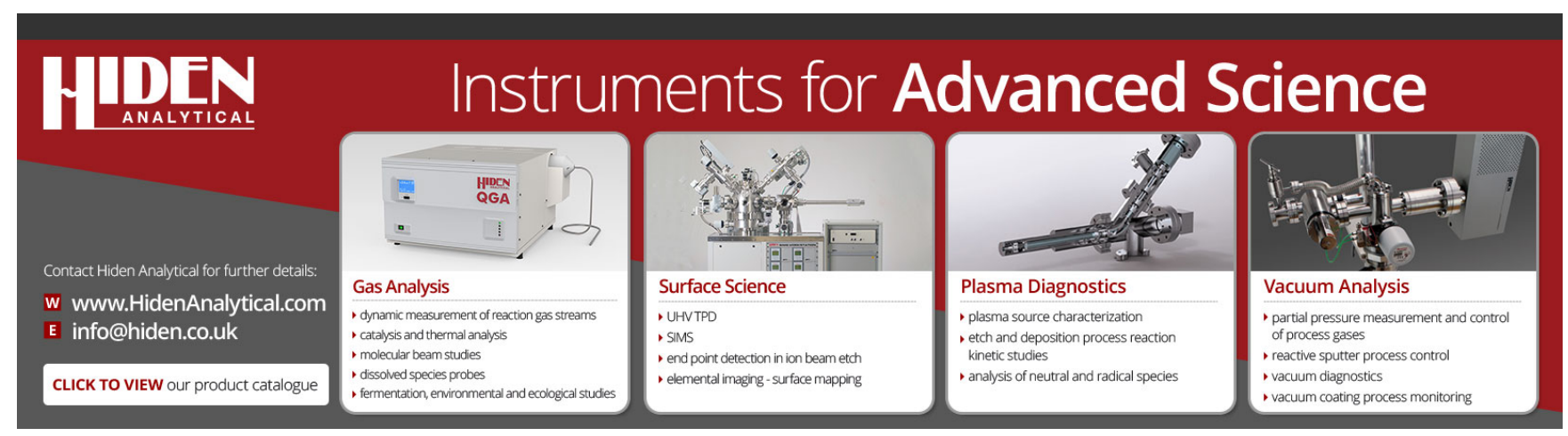




\title{
Nitrogen acceptor in 2H-polytype synthetic $\mathrm{MoS}_{2}$ assessed by multifrequency electron spin resonance
}

\author{
Ben Schoenaers, Andre Stesmans, ${ }^{\text {a) }}$ and Valery V. Afanas'ev \\ Department of Physics and Astronomy, University of Leuven, 3001 Leuven, Belgium
}

(Received 11 April 2018; accepted 7 June 2018; published 9 July 2018)

\begin{abstract}
Electron spin resonance (ESR) study on 2H-polytype synthetic $\mathrm{MoS}_{2}$ revealed the $\mathrm{N}$ acceptor dopants as being characterized by a spectrum of axial symmetry $\left[g_{\|}=2.032(2) ; g_{\perp}=2.270(2)\right]$, typical for a hole-type center in $\mathrm{MoS}_{2}$. The $\mathrm{N}$ impurities substitute for $\mathrm{S}$ sites, with a density of $\sim 2.3 \times 10^{17} \mathrm{~cm}^{-3}$, which accounts for the overall p-type doping. With respect to measurements for the applied magnetic field directed along the $c$-axis, the signal consists of a ${ }^{14} \mathrm{~N}$ primary hyperfine triplet of splitting constant $A_{\|}=14.7 \pm 0.2 \mathrm{G}$ superimposed on a correlated Gaussian single central line of peak-to-peak width $\Delta B_{p p}=15.3 \pm 0.5 \mathrm{G}$, the latter making up only $\sim 26 \%$ of the total signal intensity. The current work extends on these results through extensive monitoring of the temperature (T) dependence of salient ESR parameters and studying the impact of thermal treatment. ESR signal saturation studies indicate a $\mathrm{N}$ acceptor spin-lattice relaxation time $T_{1}(4.2 \mathrm{~K}) \approx 3 \times 10^{-4} \mathrm{~s}$, notably different from the much smaller As acceptor's $T_{1}$ in geological $\mathrm{MoS}_{2}$. Concerning the thermal stability of the dopant, the $\mathrm{N}$ acceptor is found to be drastically passivated when exposed to $\mathrm{H}_{2}$ at $\sim 500{ }^{\circ} \mathrm{C}$. Yet, subsequent reactivation attempts in vacuum at temperatures up to $740{ }^{\circ} \mathrm{C}$ appear unsuccessful, urging great caution with conventional forming gas treatments at $T \gtrsim 500^{\circ} \mathrm{C}$. Combination of careful K- and Q-band ESR monitoring of the $T$-dependent signal intensity resulted in the consolidation of the $\mathrm{N}$ dopant as a shallow acceptor of activation energy $E_{a}=45 \pm 7 \mathrm{meV}$. The consolidated results establish $\mathrm{N}$ as a promising candidate for stable covalently bonded p-type doping of $\mathrm{MoS}_{2}$ layers intended for application in novel nanoelectronic devices. Published by the AVS. https://doi.org/10.1116/1.5034447
\end{abstract}

\section{INTRODUCTION}

Two-dimensional (2D) nanomaterials have arisen as increasingly appealing for pushing the boundaries of current semiconductor technology due to compelling features like the emergence of new physical properties in the transition from bulk to $2 \mathrm{D}$ materials and the promising potential to create a multitude of innovative device structures. ${ }^{1-6}$ Among the 2D materials, layered transition metal dichalcogenides (TMDs) experience an ever-growing research interest as these could potentially replace traditional semiconductors in next generation nanoelectronic devices. ${ }^{1-3,5-7}$

Among the extensive group of TMDs, molybdenite $\left(\mathrm{MoS}_{2}\right)$ has raised particular research interest: it is a strong, flexible, and atomically thin 2D layered semiconductor with a direct bandgap of $\sim 1.85 \mathrm{eV}$ in covalently bonded S-Mo-S monolayer form and an indirect bandgap of $\sim 1.3 \mathrm{eV}$ in bulk form. ${ }^{1,2}$ Moreover, due to the interlayer van der Waals bonding nature of TMD sheets, multimaterial stacking without lattice mismatch issues is made possible, hence enabling the combination of different optical, mechanical, and electrical properties to potentially create innovate devices with augmented functionality. ${ }^{8}$ The unique material properties of TMDs, and $\mathrm{MoS}_{2}$ in particular, make them remarkably suitable for application in integrated circuits and optoelectronic devices, like solar cells, and flexible and transparent electronics. ${ }^{1,2}$

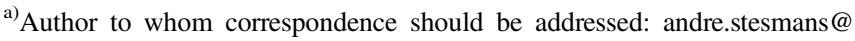
kuleuven.be
}

Obviously, full exploitation of the outstanding properties of thin $\mathrm{MoS}_{2}$ layers in novel TMD-based devices, including bipolar structures, will require the ability to properly control doping characteristics like dopant concentration, type (n or p), and stability, the latter ideally achieved by covalent bonding of substitutional impurities. Without doubt, large scale application of TMDs in novel devices is to be enabled through dependable and thoroughly controlled manufacturing of synthetic 2D materials. Currently, though, the highest mobility devices incorporating TMDs are realized by making use of micromechanically exfoliated high-quality pristine $2 \mathrm{D}$ $\mathrm{MoS}_{2}$ flakes. ${ }^{3,9,10}$ However, the experimentally established knowledge on reliable chemical doping is still inadequate, specifically regarding the unambiguous dopant identification, quantification, and energy level positioning within the $\mathrm{MoS}_{2}$ bandgap.

While not left indifferent in the past, the research interest-both theoretically and experimentally-in the understanding and realization of appropriate, well steered doping of $\mathrm{MoS}_{2}$, in 2D as well as in 3D form, has geared up steadily over the recent years, the effort being accelerated by the strive for 2D applications. First principle calculations using density functional theory have been carried out for various impurities in monolayer $1 \mathrm{H} \mathrm{MoS}$ (Refs. 11-19) and bulk $\mathrm{MoS}_{2},{ }^{13,19-22}$ and in various works the nature of chemical dopants has been investigated experimentally, ${ }^{21,23-33}$ with several studies focusing on the realization of a $2 \mathrm{D}$ diluted magnetic semiconductor through doping by transition metals. ${ }^{29,30}$ In general, the experimental studies concur that 
adequate n-type doping of $\mathrm{MoS}_{2}$ can be achieved by incorporation of Re impurities substituting for $\mathrm{Mo},{ }^{27}$ well corroborated by theoretical insight. ${ }^{11,13}$ In a separate work on effectuating low-resistivity metal-semiconductor contacts, high n-type doping of few-layer exfoliated $\mathrm{MoS}_{2}$ flakes has been obtained by means of a molecular doping technique (soaking in 1,2-dichloroethane), the doping mechanism being tentatively ascribed to $\mathrm{Cl}$ atoms occupying preexisting $\mathrm{S}$ vacancies. ${ }^{33}$

On the other hand, chemical p-type doping has already been demonstrated back in the 1970s, based on electron spin resonance (ESR) studies, establishing substitutional $\mathrm{Zr}$, Nb, $\mathrm{As}$, and $\mathrm{V}$ in $3 \mathrm{R} \mathrm{MoS}_{2}$ grown by chemical vapor transport $^{23,24}$ and As in natural $2 \mathrm{H}-\mathrm{MoS}_{2}$ (Ref. 24) to behave as acceptors. Regarding $\mathrm{Nb}$, the p-type behavior has recently been confirmed experimentally ${ }^{25,26}$ and corroborated theoretically. ${ }^{11}$ A recent low-temperature $(T)$ ESR study has demonstrated As to behave as a very shallow acceptor with activation energy $E_{a}=0.7 \pm 0.2 \mathrm{meV}$ in natural $2 \mathrm{H}-\mathrm{MoS}_{2},{ }^{31}$ and in related ESR work on the same material, it has been concluded that $\mathrm{Pb}$ impurities operate as acceptors, ${ }^{28}$ with $\mathrm{Pb}$ likely substituting for Mo.

Additionally, p-type doping by $\mathrm{P}$ ion implantation was demonstrated for field effect transistors with $\sim 20 \mathrm{~nm}$ thick channels of exfoliated $\mathrm{MoS}_{2},{ }^{21}$ and a remote $\mathrm{N}_{2}$ plasma surface treatment has also been put forward by a recent study as an efficient strategy for p-type nitrogen doping of exfoliated $\mathrm{MoS}_{2}$ flakes. ${ }^{32}$ Remarkably, other work from the same group concluded that geological $\mathrm{MoS}_{2}$ may even incorporate both $\mathrm{n}$ - and p-type regions within a single sample. ${ }^{34}$ Finally, very recent work $^{35}$ has reported on p-type doping resulting from $\mathrm{MoS}_{\mathrm{x}} \mathrm{O}_{3-\mathrm{x}}$ inclusions introduced in $\mathrm{MoS}_{2}$ films (1.5 $\mathrm{nm}$ thick) grown by physical vapor deposition after exposure to oxygen.

In recent ESR work, ${ }^{36,37}$ we reported on the observation and identification of the $\mathrm{N}$ acceptor in $2 \mathrm{H}-\mathrm{MoS}_{2}$, the N substituting for an $\mathrm{S}$ site. In extending on these results and with the aim to further fundamental insight, we here present the results of an extensive multifrequency ESR study carried out over three observational frequencies and a wide $T$ range, in combination with thermal treatment in $\mathrm{H}_{2}$ and vacuum. Besides basic ESR spectral characterization, attention is focused on the dopant's thermal stability and consolidation of the thermal activation energy. The nitrogen dopant, substituting for $\mathrm{S}$, is reliably established as an adept shallow and stable substitutional acceptor.

\section{ESR ON N ACCEPTOR IN MOS 2 : SYNOPSIS}

In overview, two ESR signals have been related with $\mathrm{N}$ impurities in synthetic $2 \mathrm{H}-\mathrm{MoS}_{2},{ }^{36}$ both exhibiting distinct anisotropy with respect to the angle $\varphi_{B}$ that the applied magnetic field, B, makes with the $c$-axis (sample main face normal $\mathbf{n}$ ). The most prominent one is illustrated in Fig. 1, showing an X-band observation made at $77 \mathrm{~K}$ for $\mathbf{B}$ along the "high-symmetry" crystal direction $\mathbf{n}$ ( $c$-axis); clearly, the signal emerges as an apparent "three-peak" structure, which, upon deeper examination, unfolds as a symmetric triplet of overlapping lines of total width $\sim 42$ G. As illustrated in

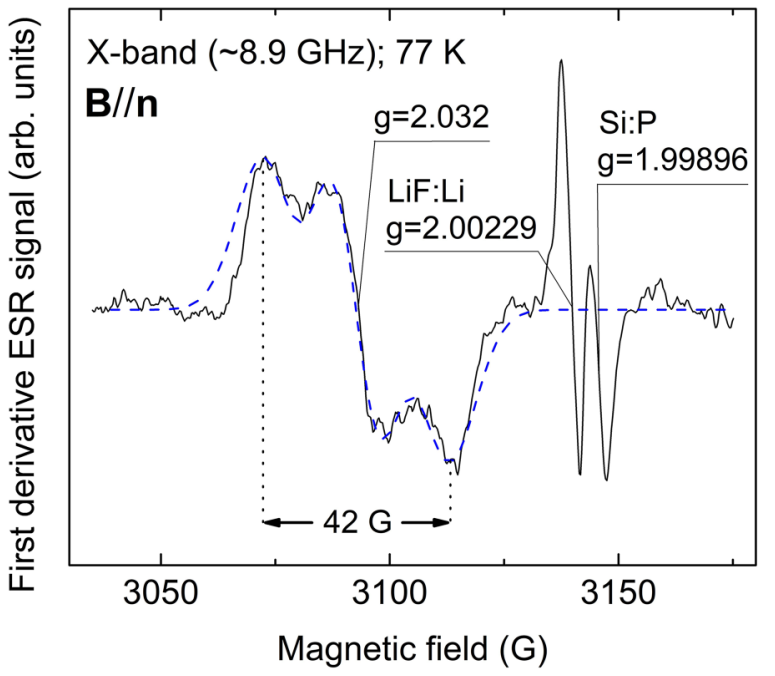

FIG. 1. X-band ESR spectrum of $\mathrm{N}$ acceptor impurities in synthetic $\mathrm{MoS}_{2}$, observed for $\boldsymbol{B} \| c$-axis. The dashed curve superposed on the spectrum represents an optimized computer simulation obtained as the sum of a 1:1:1 hf triplet of isoshaped equally intense lines $\left(\Delta B_{p p}=13.6 \pm 0.5 \mathrm{G}\right.$; Gaussian shape) superimposed on a central line $\left(\Delta B_{p p}=15.3 \pm 0.5 \mathrm{G}\right.$; intensity $\sim 26 \%$ of the total spectrum; Gaussian shape) centered at (close to) the same $g=$ $2.032 \pm 0.002$. The small signals emerging at $g=2.00229$ and $g=1.99896$ originate from co-mounted marker samples LiF:Li and Si:P, respectively.

Fig. 2(b), a virtually identical spectrum, regarding both shape and width, is recorded at K-band frequency, which has led to the conclusion of the triplet structure to originate from hyperfine (hf) interaction of the unpaired electron spin with a single nucleus of spin $I=1$; the alternative possibility for the overall signal structure to be of more powder pattern nature is ruled out.

Computer-assisted analysis indicates that the $\mathbf{B} / / \mathbf{n}$ spectrum may be appropriately fitted [dashed curves in Figs. 1 and 2(b)] as a weighed sum of an isoshaped (Gaussian; $\left.\Delta B_{p p}=13.6 \pm 0.5 \mathrm{G}\right) 1: 1: 1$ triplet of hf splitting constant $A_{/ /}$ $=14.7 \pm 0.2 \mathrm{G}$ superimposed on a single central line $(\mathrm{CL})$ of $\Delta B_{p p}=15.3 \pm 0.5 \mathrm{G}$ (Gaussian), both centered at (close to) the same $g$ value $\left(g_{\|}=2.032\right)$. The triplet and CL shift in tandem as $\varphi_{B}$ is varied, indicating a common defect origin. The totality of the spectrum corresponds to an impurity density of $(2.3 \pm 0.2) \times 10^{17} \mathrm{~cm}^{-3}$, of which the CL accounts for $\sim 26 \%$. The latter density appears uniform over the $\mathrm{mm}$-size scale (2-7 mm main sample size).

Guided by further analysis, the main triplet has been assigned as a single ${ }^{14} \mathrm{~N}$ hf splitting originating from isolated $\mathrm{N}$ impurities in $\mathrm{MoS}_{2}$. Furthermore, the signal is found to exhibit axial symmetry characterized by $g_{\| /}=2.032 \pm 0.002$ and $g_{\perp}=2.270 \pm 0.002$ - a kind of anisotropy, i.e., magnitude of $g\left(>g_{e}=2.00232\right.$, the free electron value $)$ and $\left|g_{\perp}-g_{\|}\right|$, and symmetry about the $c$-axis, typical for covalently bonded substitutional impurities in $\mathrm{MoS}_{2}$ behaving as acceptors, as previously established for As (substituting for $\mathrm{S}$ ), ${ }^{24,31} \mathrm{Nb}$, $\mathrm{Zr}$, and $\mathrm{V}^{23}$ This has led to the assignment of the observed ESR signal to $\mathrm{N}$ acceptors substituting for $\mathrm{S}$ vacancies, in line with previous theoretical insight. ${ }^{11,12}$

For illustrative reasons, a more in-depth spectroscopic exposure of the explicit anisotropy of the main $\mathrm{N}$ impurity signal, 

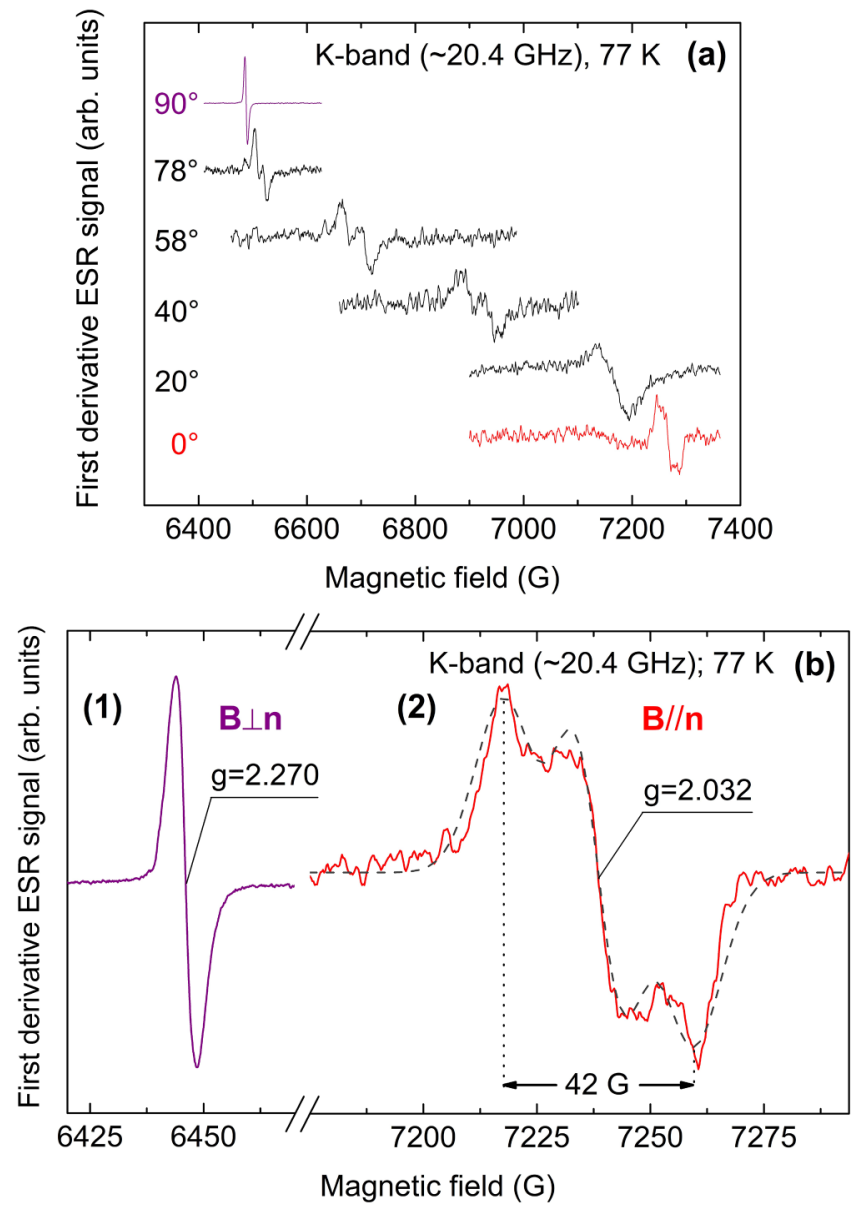

FIG. 2. (a) K-band ESR spectra of the $\mathrm{N}$ impurity observed at $77 \mathrm{~K}$ at various values of $\varphi_{B}$ for $\mathbf{B}$ rotating over an angle of $90^{\circ}$ with respect to the $c$-axis. The signals for $\varphi_{B}=0^{\circ}$ and $90^{\circ}$ are characterized by $g_{\perp}=2.270 \pm$ 0.002 [spectrum (1)] and $g_{\| /}=2.032 \pm 0.002$ [spectrum (2)], respectively. The hf triplet and CL appear to shift in tandem, indicating a common defect origin and, notably, the hf triplet, of total width $\sim 42 \mathrm{G}$ as indicated by the dotted lines, observed for $\mathbf{B} / / \mathbf{n}$ is narrowed down to become enclosed in a single, seemingly structureless, intense signal of peak-to-peak width $\Delta B_{p p}=$ $4.8 \pm 0.2 \mathrm{G}$ for $\varphi_{B} \rightarrow 90^{\circ}(\mathbf{B} \perp \mathbf{n})$, suggesting significant anisotropy of the hf matrix. (b) A zoomed-in comparison of the $\varphi_{B}=0^{\circ}$ and $\varphi_{B}=90^{\circ}$ responses observed over narrow field scans. The dashed curve superposed on spectrum (2) represents an optimized computer simulation obtained as the sum of a $1: 1: 1 \mathrm{hf}$ triplet of isoshaped equally intense lines superimposed on a central line centered at (close to) the same $g=2.032 \pm 0.002$.

regarding both shape and $g$ value, is provided in Fig. 2(a) showing the main $\mathrm{N}$ signal recorded at $77 \mathrm{~K}$ (K-band) for six different angles $\varphi_{B}$ ranging from $0^{\circ}$ to $90^{\circ}$ (corresponding to $\mathbf{B} / / \mathbf{n}$ and $\mathbf{B} \perp \mathbf{n}$, respectively). Clearly, as mentioned before, the hf triplet and the central line tightly shift in tandem, indicating a common defect origin. Also, it appears that the hf splitting matrix possesses a relatively high level of anisotropy as the hf triplet strongly narrows down to become comprised within a single, seemingly structureless, intense peak of peak-to-peak width $\Delta B_{p p}=4.8 \pm 0.2 \mathrm{G}$ for $\varphi_{B} \rightarrow 90^{\circ}(\mathbf{B} \perp \mathbf{n})$. The strikingly strong anisotropy of the overall signal shape is fully exposed in "enlarged view" in Fig. 2(b), showing a comparison of narrow field sweep K-band spectra observed at $77 \mathrm{~K}$ for the "extreme" field orientations, i.e., $\mathbf{B} \perp \mathbf{n}$ and $\mathbf{B} / / \mathbf{n}$ [signal (1) and (2), respectively].
In analogy with previous work, ${ }^{38,39}$ the CL part of the total spectrum suggests some nonuniform doping with $\sim 26 \%$ of the $\mathrm{N}$ dopants not residing in the preferred "isolated" doping state. Indeed, similar to the case of n-type $\mathrm{P}$ doping in $\mathrm{Si}$, the appearance of the central line would correspond to exchange coupled electrons at clustered dopant impurities (mostly pairs) resulting in averaging out of the ${ }^{14} \mathrm{~N}$ hf splitting with the electrons now acting as one unit. ${ }^{38,39}$ This interpretation is further corroborated by the observed second ESR signal, i.e., a correlated (i.e., regarding anisotropy and signal shape) single half field line (characterized by $g_{\perp}=3.92 \pm 0.01, \Delta B_{p p}=9 \pm 1 \mathrm{G}$ at $4.2 \mathrm{~K}$ for $\mathbf{B} \perp \mathbf{n}$, and an intensity of $\sim 0.6 \%$ of the main signal) indicative of the presence of $S \geq 1$ centers ( $\mathrm{N}$ dopant clusters).

Finally, it should be noticed that, since no particular doping process has been applied, the incorporation of $\mathrm{N}$ impurities must be sample growth related.

\section{EXPERIMENTAL DETAILS}

\section{A. Samples}

The examined specimen is a commercial bulk synthetic 2H-polytype $\mathrm{MoS}_{2}$ crystal of, as specified by the supplier, ${ }^{40}$ $99.995 \%$ purity, grown by chemical vapor deposition (CVD) using high-purity Mo and sulfide sources. A thermoelectric test demonstrated the sample to be manifest p-type. From the mother crystal, thin $(\sim 0.2-0.4 \mathrm{~mm})$ ESR-appropriate slices of $\sim 2 \times 7 \mathrm{~mm}^{2}$ main area were cut, with the $c$-axis along $\mathbf{n}$.

Measurements were carried out on samples in the as-received state and after thermal treatment in $\mathrm{H}_{2}(\sim 1.1 \mathrm{~atm})$ and vacuum $\left(<7 \times 10^{-6} \mathrm{mbar}\right)$ for $\sim 45 \mathrm{~min}$ at selected temperatures in the range $22-500{ }^{\circ} \mathrm{C}$ and $500-740{ }^{\circ} \mathrm{C}$, respectively, in order to assess the reactivity and thermal stability of observed ESR-active centers. More specifically, as alluded to in the previous section, the focus is on the $\mathrm{N}$ impurities, operating as acceptors. Thermal treatments were performed using a conventional resistively heated furnace system foreseen with a doublewalled high-purity quartz insert, as described elsewhere. ${ }^{41}$

With applying thermal treatments in $\mathrm{H}_{2}$, the main goal is the investigation of the potential inactivation (passivation) of the acceptors through binding to $\mathrm{H}$ [an effect well known for some dopants in other semiconductors (see, e.g., Ref. 42)]. In a next sequence, this is followed by the analysis of their potential reactivation (acceptor-hydrogen dissociation) under annealing in vacuum, herewith, at the same time, intending to provide useful information on the overall thermal stability - as functional dopants - of the substitutional covalently bonded acceptors. The thermal treatment analysis was carried out by applying a series of thermal steps sequentially on one sample, at incremental $T$, in order to expose the evolution of the thermal impact, and potentially, reveal any abrupt transitions.

Additionally, it may be useful to add that over all the thermal treatments applied, no creation or activation of additional "new" ESR signals was noticed.

\section{B. ESR spectroscopy}

Conventional continuous-wave absorption first derivative $\mathrm{X}$ ( $\sim 8.9 \mathrm{GHz}), \mathrm{K}-(\sim 20.4 \mathrm{GHz})$, and Q-band $(\sim 34.3 \mathrm{GHz})$ ESR 
measurements were carried out at temperatures ranging from 4.2 to $298 \mathrm{~K}$ using periodic modulation, $B_{m} \cos \left(\omega_{m} t\right)$, at $\omega_{m} /$ $2 \pi \approx 100 \mathrm{kHz}$ of $\mathbf{B}$. The accurate determination of defect densities and $g$ values of the observed signals was aided by employing two co-mounted calibrated marker samples, i.e., LiF:Li $(g=2.002293 \pm 0.000002 ; \text { spin } S=1 / 2)^{43}$ and $\mathrm{Si}: \mathrm{P}[g(4.3 \mathrm{~K})=$ $1.99869 \pm 0.00002 ; S=1 / 2]$. ${ }^{44}$ Defect densities were reliably obtained by double numerical integration of the observed $\mathrm{d} P_{\mu r}$ l $\mathrm{d} B$ signals ( $P_{\mu r}$ the reflected microwave power) where care was taken to record spectra under distortion-avoiding spectrometer settings, i.e., appropriately restricted $B_{m}$ and incident microwave power $P_{\mu}$ (see, e.g., Ref. 45 for spectroscopic details). The signal anisotropy was monitored by varying the angle $\varphi_{B}$ over a range of $\sim 180^{\circ}$.

\section{RESULTS AND DISCUSSION}

\section{A. ESR spectrum}

\section{Temperature dependence}

Aside from the highly anisotropic overall signal shape, as aforementioned, the observational temperature also strongly
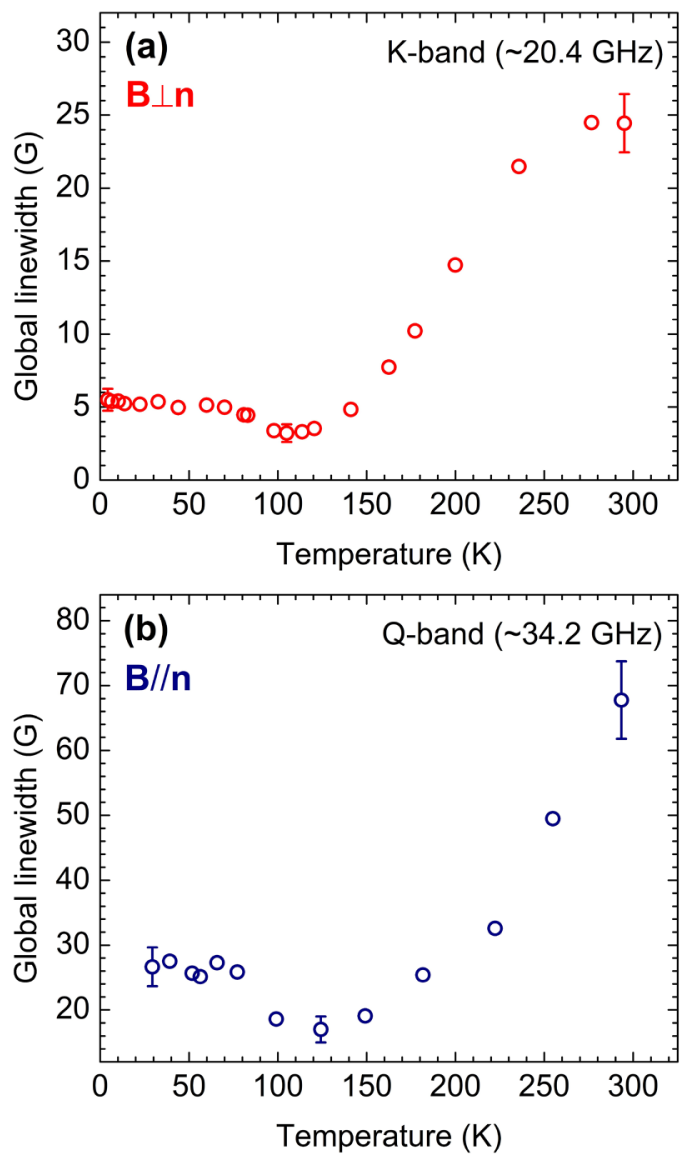

affects the appearance of the $\mathrm{N}$ acceptor signal, as illustrated in Fig. 3, showing K- [panel (c)] and Q-band [panel (d)] spectra recorded for $\mathbf{B} \perp \mathbf{n}$ and $\mathbf{B} / / \mathbf{n}$, respectively, over a broad $T$ range $(4.2-298 \mathrm{~K})$. A similar trend of signal broadening with increasing $T$, which can be primarily ascribed to a raising spin-lattice interaction ( $T_{1}$ relaxation), is observed for both orientations, reaching strong line broadening at high temperatures $(T \gtrsim 200 \mathrm{~K})$. As a result, with increasing temperature in this $T$ range, the observability (signal-to-noise ratio) of the ESR signal is gradually reduced, which is further lowered due to the expected Curie-type susceptibility behavior, $\chi \propto 1 / T$, and the decreasing amount of neutral, ESR-active, acceptors due to dopant activation. With raising $T$, these mechanisms make it thus gradually more troublesome to reliably distinguish the targeted signal from relatively "growing" omnipresent (cavity) background responses as illustrated in Fig. 3(d) (Q-band observations): Here, for one, the hf pattern (first, second, and third lines) of ${ }^{55} \mathrm{Mn}$ isotope $(I=5 / 2)$ impurities is seen to come out relatively more prominent with increasing $T$. As a result, when ESR monitoring the density of activated dopants (see below), this obfuscating trend will require an especially cautious double
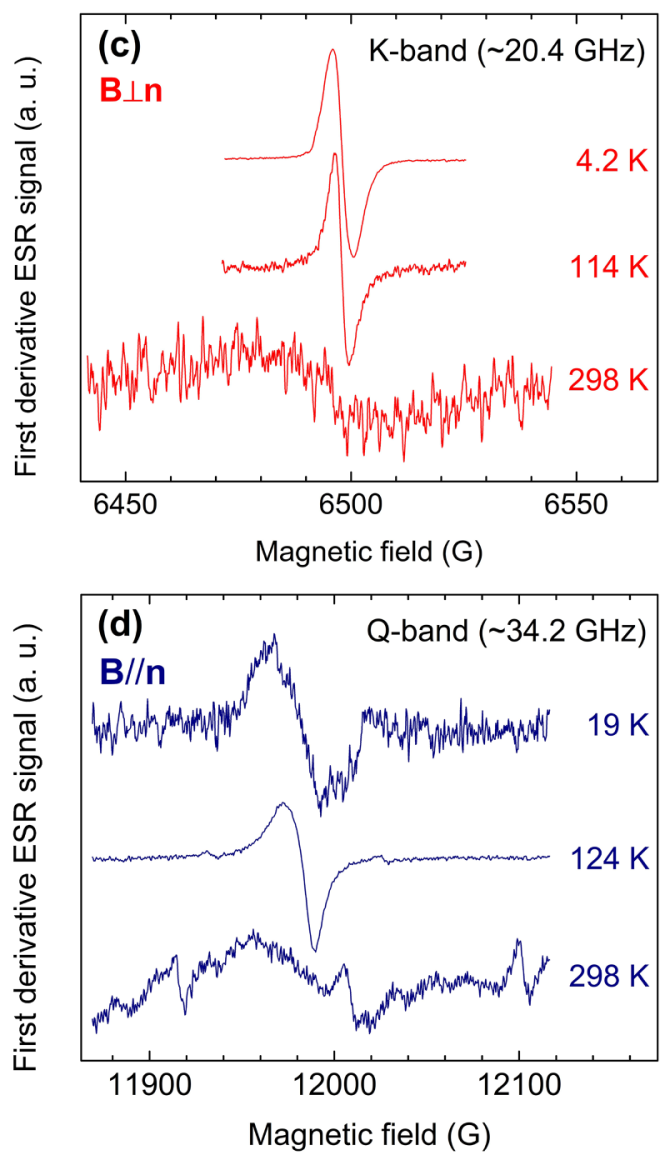

FIG. 3. Temperature dependence of the global linewidth of the first derivative K-band (a) and Q-band (b) N acceptor signal observed in as-received synthetic $2 \mathrm{H} \mathrm{MoS}{ }_{2}$ for $\mathbf{B} \perp \mathbf{n}$ and $\mathbf{B} / / \mathbf{n}$, respectively, over the $T$ range 4.2-298 K. Starting from a plateau value and remaining constant for $T \lesssim 75 \mathrm{~K}$, the linewidth reaches a minimum value of $\Delta B_{p p}=3.2 \pm 0.2 \mathrm{G}$ at an intermediate temperature of $\sim 105 \mathrm{~K}$ for $\mathbf{B} \perp \mathbf{n}$ (a), while for $\mathbf{B} / / \mathbf{n}$ (b) a minimum total linewidth of $\Delta B_{p p}=$ $17 \pm 2 \mathrm{G}$ is achieved at a similar $\sim 125 \mathrm{~K}$. For higher temperatures, the signal width is seen to grow fastly. The evolution of the signal shape of the K-band $(\mathbf{B} \perp \mathbf{n})$ and Q-band $(\mathbf{B} / / \mathbf{n}) \mathrm{N}$ acceptor ESR spectra is shown in (c) and (d), respectively. The seemingly structureless single signal for $\mathbf{B} \perp \mathbf{n}$ at low $T$ undergoes distinct broadening with increasing $T$, ascribed to the raising spin-lattice interaction ( $T_{1}$ relaxation). A similar signal broadening is observed for both orientations, as expected. Additional background signals, such as the hf pattern (first, second, and third lines) of ${ }^{55} \mathrm{Mn}(I=5 / 2)$ cavity background impurities gradually arise more prominently in the Q-band spectra (d) due to the severe broadening and, accordingly, apparent fading of the $\mathrm{N}$ signal at high $T$. 
numerical integration routine to obtain trustworthy data at temperatures approaching room temperature.

The detailed $T$ dependence of the linewidth at K-band for $\mathbf{B} \perp \mathbf{n}$, shown in Fig. 3[panel (a)], exposes a minimum peak-to-peak linewidth of $\Delta B_{p p}=3.2 \pm 0.2 \mathrm{G}$ reached at $\sim 105 \mathrm{~K}$. Similarly, the $T$ dependence of the global (total) linewidth at Q-band for $\mathbf{B} / / \mathbf{n}$ is shown in Fig. 3(b), revealing a minimum total linewidth of $\Delta B_{p p}=17 \pm 2 \mathrm{G}$ at $\sim 125 \mathrm{~K}$. As exposed, the trend is similar for both cases: Starting from the low- $T$ end, the width remains constant (plateau value) up to $\sim 75 \mathrm{~K}$, to narrow down and reach a minimum at $\sim 105-125$ $\mathrm{K}$ from whereon the signal steadily broadens fastly with raising $T$. The temperature at which the minimum linewidth is reached seems similar for both orientations, despite the linewidth being slightly more precarious to determine at Q-band for $\mathbf{B} / / \mathbf{n}$ due to an, albeit modestly, changing overall signal shape. The observation of a minimum linewidth at an intermediate temperature is indicative of the presence of one (or more) broadening mechanisms in addition to the common $T_{1}$ relaxation. Further investigation on the appropriate relaxation mechanisms is set aside for future work.

\section{Saturation}

Another useful spectral characteristic that may assist in defect identification is the ESR saturability. In the search for similarities, the saturability of the current $\mathrm{N}$ main signal has been compared to that of the previously studied As signal in geological $\mathrm{MoS}_{2}{ }^{31}$ Continuous-wave saturation curves of both signals were recorded by inserting two $\mathrm{MoS}_{2}$ samples, i.e., the geological (As containing) and synthetic ( $\mathrm{N}$ dopant) one, in the spectrometer cavity together with some crushed HSQ200-type fused quartz ${ }^{46}[[\mathrm{OH}]<30$ ppm (other impurities) $<20 \mathrm{ppm}]$ and, for reference, a Si:P marker. ${ }^{44}$

Figure 4 shows the derivative amplitude $A_{p p}$, normalized in the nonsaturation regime, i.e., $B_{1}<10^{-4} \mathrm{G}$, for each ESR signal as a function of $B_{1}$, the amplitude of the magnetic microwave field at the sample position in the cavity, derived from the incident microwave power $P_{\mu}$ and quality factor $Q$. The data are mutualy normalized in the nonsaturation regime, i.e., $B_{1}<10^{-4}$ G. All measurements were performed at $4.2 \mathrm{~K}$ with $\mathbf{B} \perp c$-axis for both $\mathrm{MoS}_{2}$ samples. Figure 4 convincingly demonstrates the distinctly different relaxation behavior of the four ESR signals with the $\mathrm{Si}: \mathrm{P}$ and $\mathrm{E}^{\prime}$ defect (generic entity $\mathrm{O}_{3} \equiv \mathrm{Si} \cdot$ ) signal, the latter stemming from the crushed fused quartz sample, exhibiting the expected previously reported behavior. ${ }^{47}$ The Si:P signal is nonsaturating over the whole $B_{1}$ range covered, while the $\mathrm{E}^{\prime}$ response shows high saturability. Pertinently, the saturation curves of the $\mathrm{N}$ and As acceptor signals are manifestly different despite their respective densities and substitutional position in the crystal lattice being similar. But this may be according to expectations. Indeed, as the saturation behavior is governed by the product $T_{1} T_{2}{ }^{48}$ with $T_{1}$ being the spin-lattice relaxation time and $T_{2}$ the spin-spin relaxation time, the stronger spin-orbit coupling of As as compared to that of $\mathrm{N}$ results in a better coupling to the lattice and, consequently, saturation sets in at a higher incident microwave power level

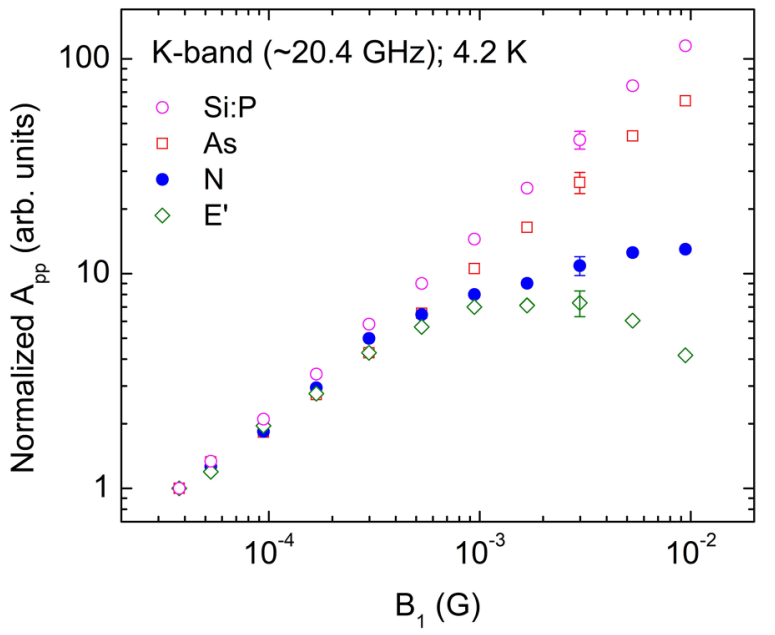

FIG. 4. Comparison of continuous-wave saturation data measured at $4.2 \mathrm{~K}$ for four ESR signals. This includes the main $\mathrm{N}$ signal measured on the current synthetic $\mathrm{MoS}_{2}$ specimen in the as-received state, the As acceptor signal from a geological $\mathrm{MoS}_{2}$ sample, the $\mathrm{E}^{\prime}$ signal in crushed fused $\mathrm{SiO}_{2}$, and, for reference, the Si:P marker signal, represented by filled circles, unfilled squares, diamonds, and circles, respectively. Both the $\mathrm{E}^{\prime}$ and Si:P marker signal (the latter not saturating) exhibit the expected saturation behavior. While the $\mathrm{N}$ acceptor's saturation curve appears to reach a maximum value at $\sim 0.02 \mathrm{G}$ corresponding to $T_{1}(4.2 \mathrm{~K}) \approx 3 \times 10^{-4} \mathrm{~s}$, the As acceptor's signal does not saturate over the $B_{1}$ range covered, indicating, for comparable signal widths, a distinctly smaller $T_{1}$ value compared to $\mathrm{N}$.

for the As acceptor signal. Therefore, the As signal data in Fig. 4 display only marginal saturation over the $B_{1}$ range covered. The exhibited resonant microwave saturation behavior is thus found in line with the assigned $\mathrm{N}$ and As acceptor signal nature.

In contrast with the As signal, the $\mathrm{E}^{\prime}$ signal shows a maximum signal height at $\sim 10^{-3} \mathrm{G}$, as expected, ${ }^{47}$ while the $\mathrm{N}$ signal tends to reach a maximum value of the saturation curve outside of the $B_{1}$ range covered. Yet, from the trend of the $\mathrm{N}$ saturation curve, the maximum value can be estimated as $B_{1} \approx 0.02 \mathrm{G}$. According to theory, ${ }^{49}$ the maximum of the saturation curve corresponds to

$$
B_{1}^{2} \gamma^{2} T_{1} T_{2} \approx \frac{1}{2},
$$

where $\gamma=g \mu_{B} / \hbar$ is the gyromagnetic ratio, with $\mu_{B}$ being the Bohr magneton and $2 \pi \hbar$ Planck's constant, and

$$
T_{2}=\frac{2}{\sqrt{3} \gamma \Delta B_{p p}}
$$

resulting in $T_{1}(4.2 \mathrm{~K}) \approx 3 \times 10^{-4} \mathrm{~s}$ for the $\mathrm{N}$ center.

\section{B. Thermal treatment: Dopant stability}

Aiming at a better understanding of dopant stability, the $\mathrm{N}$ acceptor was subjected to two series of thermal treatments, one first performed in $\mathrm{H}_{2}$ (1.1 atm), and the other in high vacuum. Thermal steps were performed sequentially on the same sample physically, priorly subjected to a "reference" 


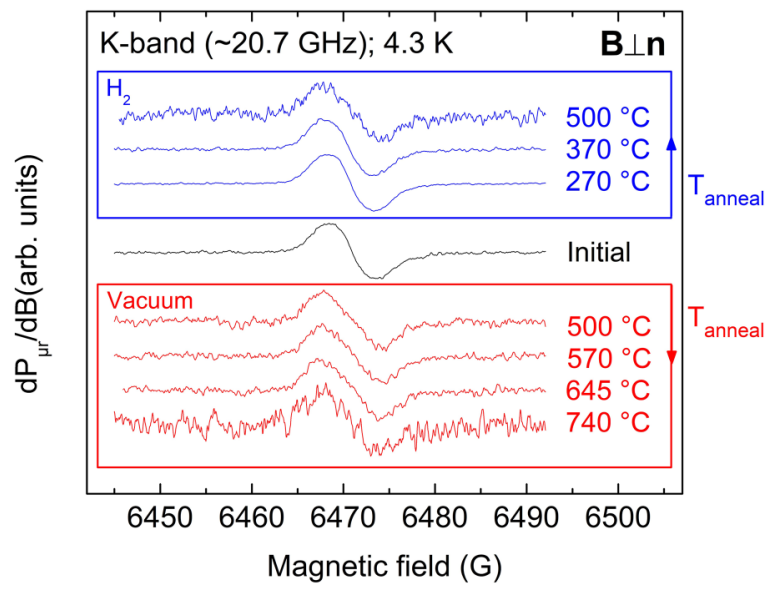

FIG. 5. Effect of applied thermal treatments in $\mathrm{H}_{2}(1.1 \mathrm{~atm} ; \sim 45 \mathrm{~min})$ and vacuum $\left(<7 \times 10^{-6}\right.$ mbar; $\left.\sim 45 \mathrm{~min}\right)$ on the $\mathrm{N}$ acceptor signal observed in synthetic $\mathrm{MoS}_{2}$ for $\boldsymbol{B} \perp c$-axis. The spectrum in the center of the figure represents the $\mathrm{N}$ impurity signal of the initial sample (as-received sample subjected to vacuum anneal at $\sim 360^{\circ} \mathrm{C}$ ). The upper spectra (upper blue rectangle) show the passivation effect in $\mathrm{H}_{2}$ ambient with increasing $T_{a n}$ while the bottom spectra (lower red rectangle) display the evolution of the signal in vacuum with increasing $T_{a n}$.

setting treatment in vacuum $\left(\sim 360^{\circ} \mathrm{C} ; \sim 2 \mathrm{~h}\right)$-referred to as the initial state. Figure 5 shows an overview of observed ESR spectra over the various thermal steps applied, whereas, in quantitative terms, Fig. 6 presents the evolution of the ESR signal intensity (non-activated $\mathrm{N}$ acceptor density). Various trends are revealed: As to thermal treatment in $\mathrm{H}_{2}$, the $\mathrm{N}$ signal is seen to remain unaffected, within experimental accuracy, for heating temperatures $\left(T_{a n}\right.$ s) up to $\sim 370^{\circ} \mathrm{C}$, to then drop drastically by 1 order of magnitude for $T_{a n}$

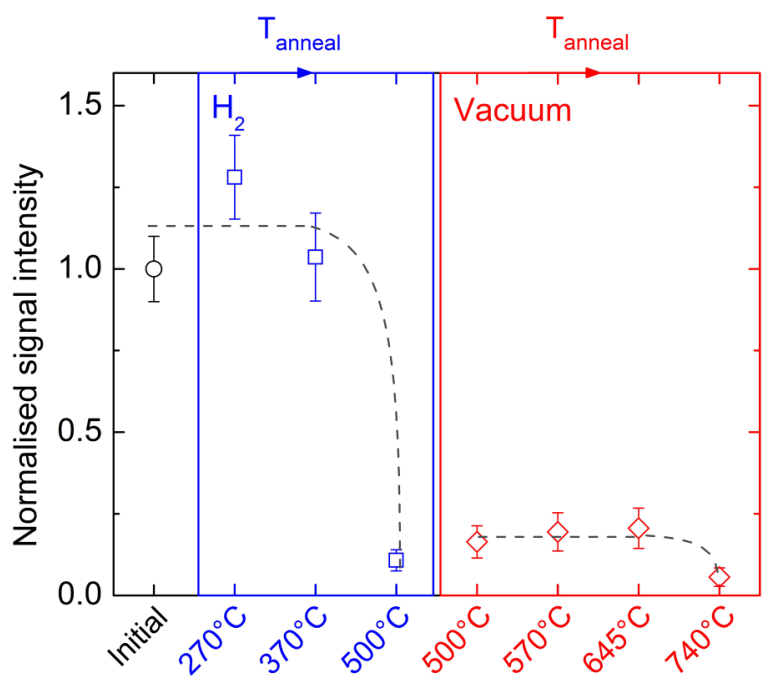

FIG. 6. Evolution of the $\mathrm{N}$ impurity signal intensity, normalized to the initial intensity value (black circle), after subsequent thermal treatments in $\mathrm{H}_{2}$ (blue squares) and vacuum (red diamonds). While the signal appears not affected (within experimental accuracy) by treatment in $\mathrm{H}_{2}$ up to $\sim 370{ }^{\circ} \mathrm{C}$, a drastic drop in signal intensity $\left(\sim 1\right.$ order of magnitude) occurs at $\sim 500{ }^{\circ} \mathrm{C}$, tentatively ascribed to passivation of $\mathrm{N}$ acceptors by bonding to $\mathrm{H}$. As the signal intensity after thermal treatments in vacuum does not increase significantly, the depassivation procedure proves to be unsuccessful. The dashed curves guide the eye. reaching $500{ }^{\circ} \mathrm{C}$. Building on previous experience with inactivation of point defects in semiconductors, such as B acceptors in $\mathrm{Si}^{42}$ this might be ascribed to direct passivation (inactivation) of $\mathrm{N}$ acceptors through defect-H bond formation.

Also guided by previous knowledge, one may then quest for possible reactivation of $\mathrm{N}$ dopants, i.e., defect- $\mathrm{H}$ bond breaking, under thermal treatment in vacuum-the intent of the second series of thermal steps applied. The data, also compiled in Fig. 6, show that, perhaps apart from a small initial increase, the vacuum treatments have little effect on the passivated $\mathrm{N}$ signal: no signal reactivation occurs up to $T_{a n}=645^{\circ} \mathrm{C}$. Along conventional interpretation [see, e.g., Ref. 50 and references therein], this failing impact of vacuum treatment may be seen as a result of strong defect-H bonding, resulting in an activation energy $\left(E_{a c t} \gtrsim 3.3 \mathrm{eV}\right)$ too high to enable any significant dopant reactivation up to $\sim 645^{\circ} \mathrm{C}$. Here, theoretical analysis and computational modeling may provide further insight.

However, further increasing $T_{a n}$ to $740{ }^{\circ} \mathrm{C}$ results in one more order of magnitude decrease in signal intensity. This "opposite," quite counterintuitive effect from vacuum treatment likely refers to fatal disruption of the substitutional $\mathrm{N}$ acceptor configuration through atomic displacement(s) and/ or complex formation with other embedded impurities or intrinsic defects, resulting in a distorted point defect of distinctly different electrical behavior.

The latter observations may expose major implications for the application of $\mathrm{N}$-doped $\mathrm{MoS}_{2}$ in novel nanoelectronic devices as once the $\mathrm{N}$ acceptor is passivated by hydrogen, it cannot be depassivated through a thermal treatment in vacuum at temperatures up to $\sim 740{ }^{\circ} \mathrm{C}$.

\section{Activation energy}

Besides serving as an exclusive technique to atomically identify and quantify a dopant impurity from its unique ESR spectrum, ESR also acts as a most useful technique to determine the dopant-type specific activation energy. An important factor determining the suitability of a dopant to be incorporated in a semiconductor material intended for novel nanoelectronic devices is its activation energy which, for an acceptor, can be written as $E_{a}=E_{A}-E_{v}$ with $E_{A}$ being the acceptor energy level and $E_{V}$ the valence band (VB) maximum. The activation energy can be reliably determined by monitoring the signal intensity, which is proportional to the ESR-active dopant density, versus $T$ together with, for analysis, the proper mathematical expression for the dopant occupation versus $T$, derived within the standard semiclassical solid state theory of band structure, as discussed before $^{31,51}$ for well-defined conditions. Additionally, it is of utmost importance in this process to take into account the intrinsic $T$ dependence of the signal intensity due to the specific magnetic behavior (susceptibility $\chi$ ) of the acceptor spin system. $^{31}$

As elaborated in previous work, ${ }^{31}$ one would preferably aim to monitor the intensity of, if emerging, the (primary) hf structure originating from and known to be distinctive for sparsely distributed decoupled dopants, the latter standing 
for the desired doping configuration, of which the corresponding spin system exhibits Curie-type susceptibility $\chi \propto 1 / T$. In this way, the impeding influence of other spectral components with a potentially unclear $T$-dependent $\chi$ is prevented. Currently, we would therefore preferably direct our attention to the intensity monitoring of only the ${ }^{14} \mathrm{~N} \mathrm{hf}$ triplet. Unfortunately, this strategy turns out to be unreliable due to considerable spectral overlap, especially for the range $T \gtrsim 100 \mathrm{~K}$. Therefore, the intensity of the entire spectrum has been monitored instead, resulting in a procedure that is nonetheless considered reasonable and acceptable as the additional CL not only makes up a rather small fraction $(\sim 26 \%)$ of the entire N ESR signal but most likely also exhibits a (close to) $\chi \propto 1 / T$ behavior, as previously demonstrated for $\mathrm{P}$ donors in $\mathrm{Si}$ at high doping levels. ${ }^{38,44}$

Directed by the approaches at hand for solid state theory based computational modeling of dopant occupation statistics, in analyzing and interpreting data, three $T$ ranges are discussed separately: First the entire $T$ range covered (4.3$298 \mathrm{~K})$, second the low- $T$ regime $(T \lesssim 150 \mathrm{~K})$, and finally, the high- $T$ data $(T \gtrsim 130 \mathrm{~K})$. All data, taken on as-received samples, have been corrected for the admitted $\chi \propto 1 / T$ dependence in the subsequent analyses.

\section{Entire $T$ range}

The obtained intensity versus $T$ plot is shown in Fig. 7 for K- [panel (a)] and Q-band [panel (b)] data. For analysis, one can derive an expression from dopant occupation statistics for the ratio of the neutral, ESR-active, acceptor density $N_{A}^{*}$ to the total acceptor density $N_{A}{ }^{31,51}$

$$
\frac{N_{A}^{*}}{N_{A}}=1-\frac{2}{1+\sqrt{1+K e^{E_{a} / k T}}},
$$

where $K=4 g_{d} N_{A} / N_{v}=\left(4 g_{d} N_{A} / N_{v 0}\right) T^{-3 / 2} \equiv K_{0} T^{-3 / 2}$, and $N_{v}=$ $2\left(2 \pi m_{v} k T / h^{2}\right)^{3 / 2} \equiv N_{v 0} T^{3 / 2}$ is the VB effective density of states, $g_{d}$ represents the impurity level spin degeneracy factor inserted for bulk $\mathrm{MoS}_{2}$ as $g_{d}=2$ since there appears to be no band degeneracy at the top of the $\mathrm{VB},{ }^{51,52} m_{v}$ is the hole effective mass, tabulated for $\mathrm{MoS}_{2}$ as $m_{v}=0.56 m_{0},{ }^{52,53}$ with $m_{0}$ being the electron rest mass, $k$ is Boltzmann's constant, and $h$ is Planck's constant. The VB effective density of states is obtained as $N_{v 0} \approx 2.02 \times 10^{15} \mathrm{~cm}^{-3} \mathrm{~K}^{-3 / 2}$. Equation (3) is valid for the conditions of doping absolutely governed by one kind of dopant with negligible compensation or intrinsic excitation, and a semiconductor nondegenerate throughout the whole $T$ range covered. It seems reasonable to assume that for the current p-type $\mathrm{MoS}_{2}$ material, the $\mathrm{N}$ acceptor is the far dominant type of dopant, appearing in a density that would dependably meet the condition of nondegeneracy, ${ }^{26}$ and supposedly also that of negligible compensation.

From optimized fitting of Eq. (3) to the K-band data in Fig. 7(a) (cf. dashed curve), we obtain $E_{a}(K)=45 \pm 7 \mathrm{meV}$, unveiling the $\mathrm{N}$ acceptor as a shallow dopant. Applying Eq. (3) to the Q-band data in Fig. 7(b) reveals an activation energy $E_{a}(Q)=50 \pm 10 \mathrm{meV}$, firmly consolidating the value obtained for K-band. Noteworthy is the comforting
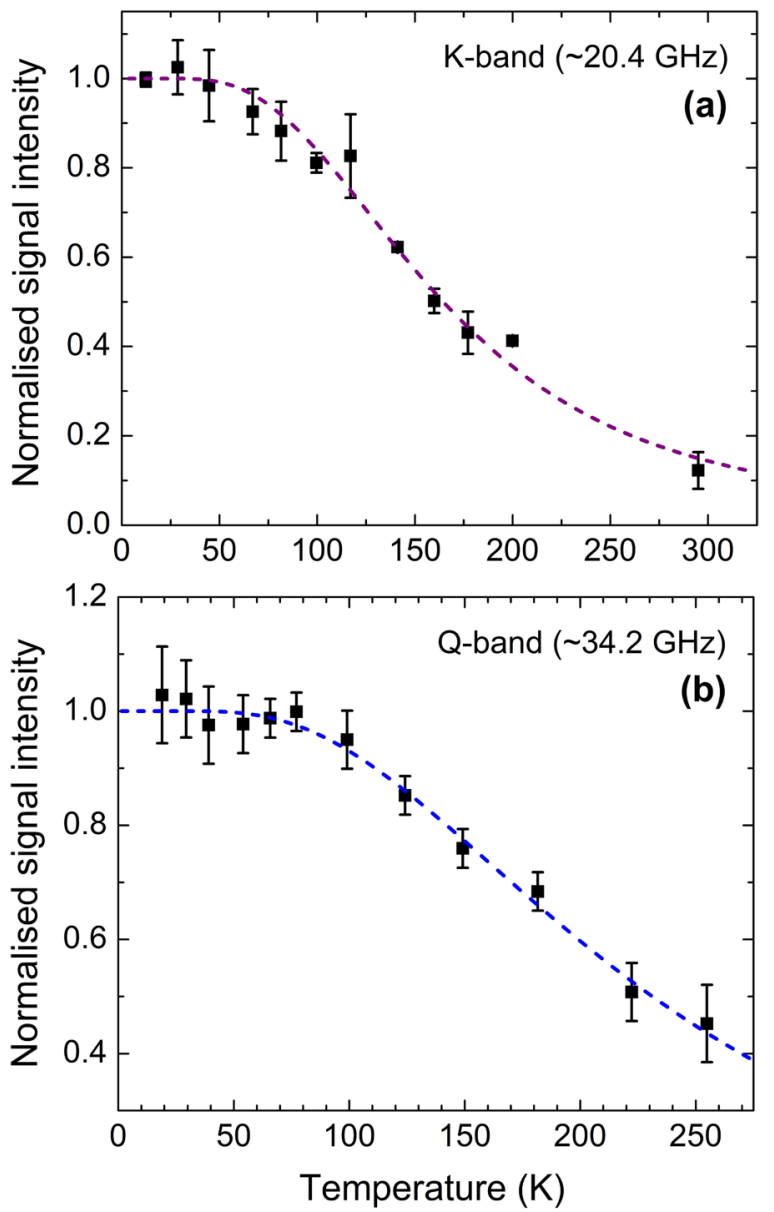

FIG. 7. Temperature dependence of the K- (a) and Q-band (b) intensity (double integral of $\mathrm{d} P_{\mu r} / \mathrm{d} B$ spectra; $\boldsymbol{B} \perp c$-axis) of the ESR signal ascribed to $\mathrm{N}$ acceptor atoms substituting for $\mathrm{S}$ in synthetic $\mathrm{MoS}_{2}$. The data, recorded over the $T$ range $4.2-298 \mathrm{~K}$, are normalized to the low- $T$ plateau value $(T \lesssim 50 \mathrm{~K})$ after correction for the admitted $\chi \propto 1 / T$ dependence. Error bars denote the spread in the data of multiple observations. The dashed curves represent an optimized fitting of Eq. (3), resulting in much similar activation energies $E_{a}=45 \pm 7 \mathrm{meV}$ and $50 \pm 10 \mathrm{meV}$ for K- and Q-band, respectively.

agreement of the emerging fitting values for the prefactors $K_{0}(K)=(7 \pm 4) \times 10^{2} \mathrm{~K}^{-3 / 2}$ and $K_{0}(Q)=(2 \pm 1) \times 10^{3} \mathrm{~K}^{-3 / 2}$, for the $\mathrm{K}$ - and Q-band analysis, respectively, with the "expected" value, $\sim 9 \times 10^{2} \mathrm{~K}^{-3 / 2}$, calculated from the values specified for $N_{v 0}, g_{d}$, and, notably, the ESR-inferred $N_{A}$ value. The $K_{0}(Q)$ value is somewhat less close. Accordingly, and within the conviction that, on spectroscopic grounds, the attained overall K-band data set on the monitoring of the $\mathrm{N}$ signal intensity versus $T$ is more accurate than the Q-band one, the inferred $E_{a}(K)=45 \pm 7 \mathrm{meV}$ is advanced as most reliable and will be quoted that way.

The self-consistency of the prefactors, together with the overall excellent fitting of the data and reassuring agreement of the K-band and Q-band results, provides confidence in the appropriateness of the applied interpretation approach [i.e., Eq. (3)]. Moreover, in combination with the value previously measured $^{31}$ for As, i.e., $E_{a}(A s)=0.7 \pm 0.2 \mathrm{meV}$, the results obtained for $E_{a}(N)$ are consistent with the theoretical prognosis for group V p-type dopants in $\mathrm{MoS}_{2}$ (Ref. 11) -albeit 
calculated for the monolayer form-in that $E_{a}(N)$ is projected to be substantially larger ( $>1$ order of magnitude) than $E_{a}(A s)$.

A final remark to be added regarding the inferred $\mathrm{N}$ acceptor activation energy is that $E_{a}$ has been obtained through monitoring the intensity of the entire $\mathrm{N}$ acceptor spectrum, i.e., the added signals of both the isolated $\mathrm{N}$ acceptors and the one, as outlined before, ascribed to clustered $\mathrm{N}$ dopants. If both species, as likely, would be characterized by somewhat dissimilar $E_{a}$ values, the inferred activation energy, advanced as pertaining to the isolated acceptors, may be affected by some averaging over both species. Yet, as the clustered part constitutes only a fraction of $\sim 26 \%$ of the total $\mathrm{N}$ signal, the influence might turn out to be rather limited.

\section{Low-T range}

Next, inspired by a conventional custom, we focused on the separate fitting of only the low- $T$ range data of the $\mathrm{N}$ signal
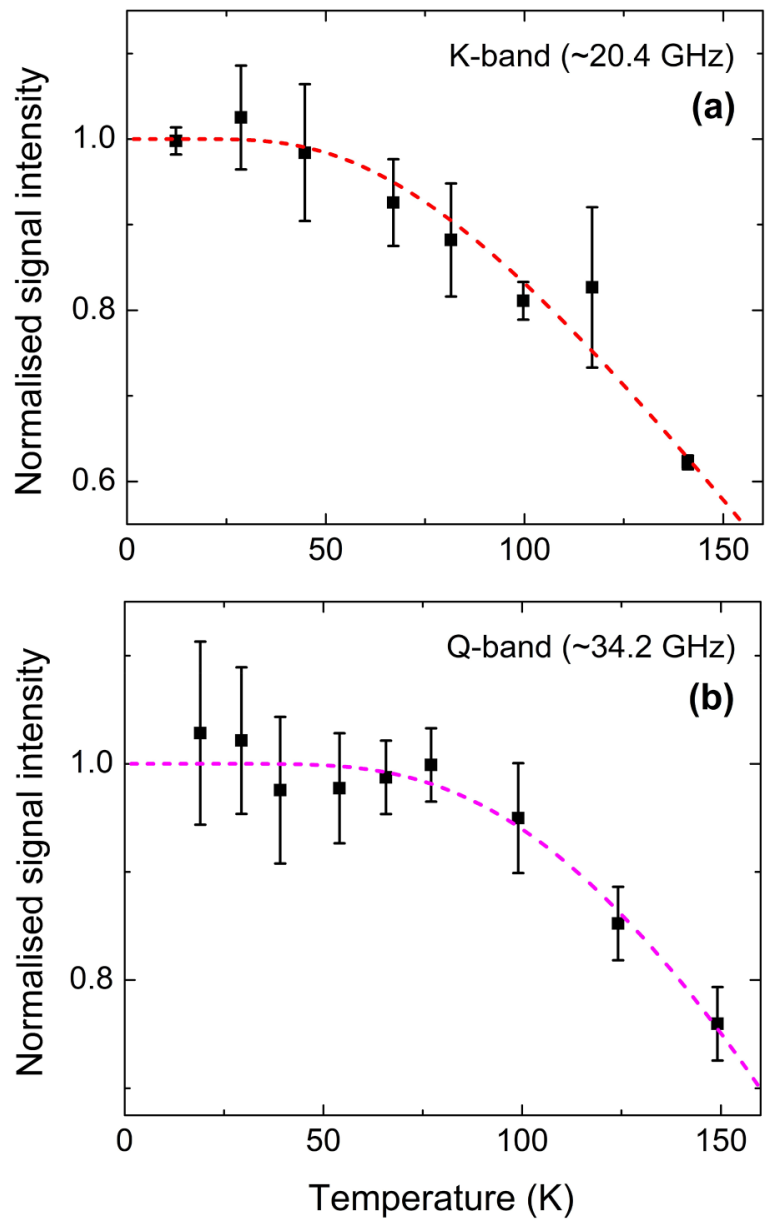

FIG. 8. Low- $T(T \lesssim 150 \mathrm{~K})$ temperature dependence of the K- (a) and Q-band (b) intensity of the $\mathrm{N}$ impurity signal observed in synthetic $\mathrm{MoS}_{2}$ for $\boldsymbol{B} \perp c$-axis. The signal intensity is normalized to the low- $T$ plateau value $(T \lesssim 50 \mathrm{~K})$ after proper correction for the admitted $\chi \propto 1 / T$ dependence. The dashed curves represent an optimized fitting of Eq. (5), resulting in an activation energy $E_{a}=33 \pm 11 \mathrm{meV}$ and $57 \pm 18 \mathrm{meV}$ for $\mathrm{K}$ - and Q-band, respectively. Error bars denote the spread in the data of multiple observations. intensity versus $T$ monitoring, presented in Fig. 8 for K- [panel (a)] and Q-band [panel (b)] measurements. For the low- $T$ condition, when relatively few holes are excited, $K \exp \left(E_{a} / k T\right) \gg 1$ (say $>10$ ), translating in the current case to $T \lesssim 150 \mathrm{~K}$, Eq. (3) approximates to

$$
\frac{N_{A}^{*}}{N_{A}}=1-\sqrt{N_{v} / g_{d} N_{A}} e^{-E_{a} / 2 k T} \equiv 1-K^{\prime} e^{-E_{a} / 2 k T},
$$

which can be rewritten as

$$
\left(1-N_{A}^{*} / N_{A}\right) T^{-3 / 4} \equiv K_{0}^{\prime} e^{-E_{a} / 2 k T},
$$

with $K_{0}^{\prime} \equiv \sqrt{N_{v 0} / g_{d} N_{A}} \cdot{ }^{31,51}$ A convenient feature of Eq. (5) is that $E_{a}$ would be directly provided by the slope of the straight line fitted to the plot $\ln \left[\left(1-N_{A}^{*} / N_{A}\right) T^{-3 / 4}\right]$ versus $1 / T .^{51}$ Fitting the K- and Q-band low- $T$ data, shown in Figs. 8 (a) and 8(b), respectively, with Eq. (5) an activation energy $E_{a}(K)=33 \pm 11 \mathrm{meV}$ is obtained for the K-band
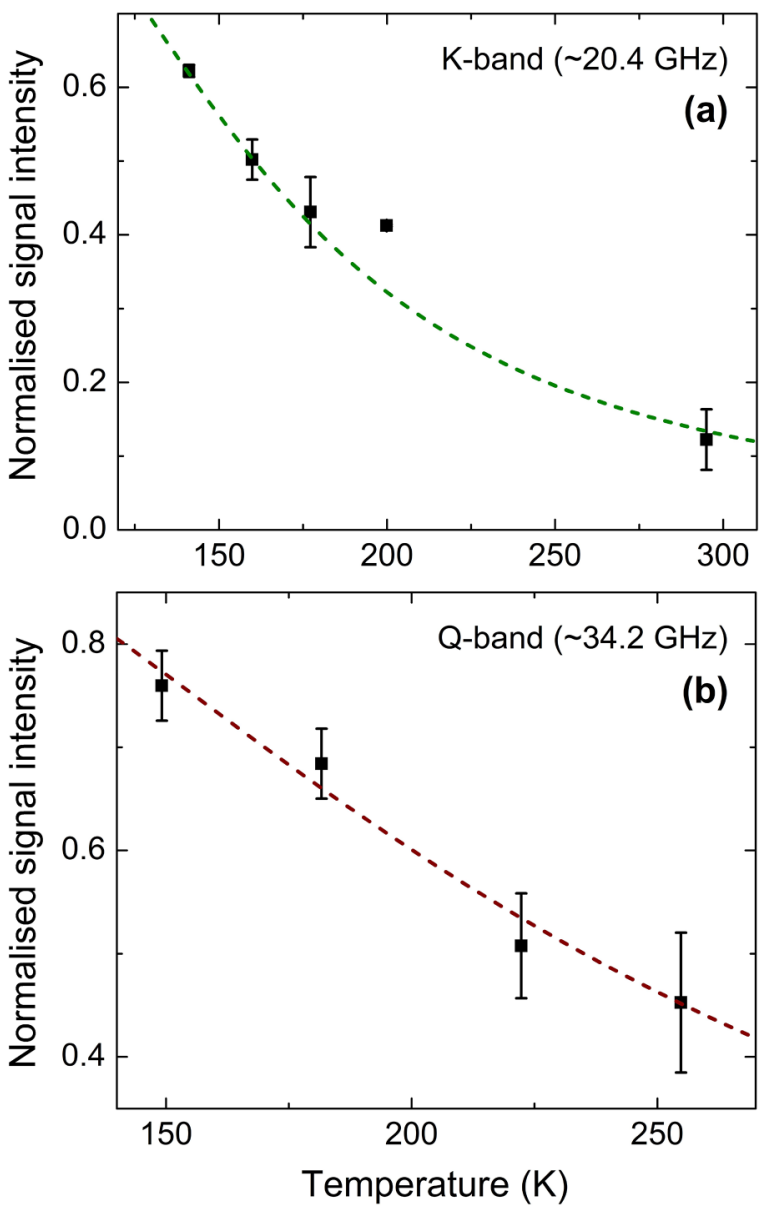

FIG. 9. High- $T(T \gtrsim 130 \mathrm{~K})$ temperature dependence of the $\mathrm{K}-$ (a) and Q-band (b) intensity of the $\mathrm{N}$ acceptor signal for $\boldsymbol{B} \perp c$-axis. The data have been normalized to the low- $T$ plateau value $(T \lesssim 50 \mathrm{~K})$ after appropriate $\chi \propto 1 / T$ dependence correction. Error bars denote the spread on the data of multiple observations. The dashed curves show an optimized fitting of Eq. (6) to the intensity data, resulting in an activation energy $E_{a}=29 \pm 12 \mathrm{meV}$ and $E_{a}=20 \pm 12 \mathrm{meV}$ for K- and Q-band, respectively. 
measurements and, in decent agreement, $E_{a}(Q)=57 \pm 18$ $\mathrm{meV}$ for the Q-band observations. Furthermore, the obtained prefactor values $K_{0}^{\prime}(K)=0.03 \pm 0.02 \mathrm{~K}^{-3 / 4}$ and $K_{0}^{\prime}(Q)=$ $0.05 \pm 0.04 \mathrm{~K}^{-3 / 4}$ for $\mathrm{K}$ - and Q-band, respectively, are both remarkably close to the value $\sim 0.07 \mathrm{~K}^{-3 / 4}$ calculated using the estimated $N_{v 0}, g_{d}$ and measured $N_{A}$. To be added here is that the obtained $E_{a}$ value from the low- $T$ data, though in fair agreement with the "overall" fitting results [cf. Eq. (3)], may be somewhat less reliable as the fitting procedures encompass fewer data points.

\section{High-T range}

Finally, in seeking further consolidation, we may address fitting of data to a separate mathematical description specifically derived for the high- $T$ part of the occupation of the acceptor dopants. ${ }^{54}$ For the usual conditions of absolute dominant doping by one kind of dopant, negligible compensation and intrinsic excitation, it has been derived that for $E_{F}-E_{A} \gg k T$ (say $\gtrsim 3 k T$, with $E_{F}$ the Fermi level)

$$
\begin{aligned}
\frac{N_{A}^{*}}{N_{A}} & =\left(1+\frac{N_{v}}{g_{d} N_{A}} e^{-E_{a} / k T}\right)^{-1} \\
& \equiv\left(1+K_{0}^{\prime \prime} T^{3 / 2} e^{-E_{a} / k T}\right)^{-1},
\end{aligned}
$$

with $K_{0}^{\prime \prime}=\left(N_{v 0} / g_{d} N_{A}\right)=\left(K_{0}^{\prime}\right)^{2} \cdot{ }^{31,51}$ Optimized fitting of Eq. (6) to the high- $T$ data $(T \gtrsim 130 \mathrm{~K})$ shown in Fig. 9 results, for the K-band data [panel (a)], in $E_{a}=29 \pm 12 \mathrm{meV}$, with $K_{0}^{\prime \prime}=(4 \pm 3) \times 10^{-3} \mathrm{~K}^{-3 / 2}$ and for Q-band [panel (b)] in a similar $E_{a}=20 \pm 12 \mathrm{meV}$, and $K_{0}^{\prime \prime}=(8 \pm 6) \times 10^{-4} \mathrm{~K}^{-3 / 2}$. The prefactor $K_{0}^{\prime \prime}$, especially for $\mathrm{K}$-band, is found to be close to the calculated value $\sim 7 \times 10^{-3} \mathrm{~K}^{-3 / 2}$, adding to the self-consistency. Once again, the obtained $E_{a}$ value from the high- $T$ data is, while still in fair agreement with the "overall" fitting result obtained via Eq. (3), deemed somewhat less reliable as the fitting routine encompasses even fewer data points than the low- $T$ analysis.

\section{SUMMARY AND CONCLUSION}

In summary, a multifrequency ESR study over the $T$ range 4.2-298 K has resulted in the identification of the $\mathrm{N}$ acceptor dopant substituting for $\mathrm{S}$ sites in $2 \mathrm{H}$-polytype synthetic bulk $\mathrm{MoS}_{2}$ crystals with a density of $(2.3 \pm 0.2) \times 10^{17} \mathrm{~cm}^{-3}$. Substantial N contamination is thus revealed as an inherent trait of the specific CVD method applied for $\mathrm{MoS}_{2}$ synthesis.

The $\mathrm{N}$ acceptor ESR spectrum is mainly composed of a primary ${ }^{14} \mathrm{~N}$ hf structure making up only $\sim 74 \%$ of the total spectrum, superimposed on a central line, revealing some nonuniform doping with $\sim 26 \%$ of the $\mathrm{N}$ dopants not residing in the preferred "isolated" doping state. The agglomeration of the $\mathrm{N}$ dopants is further supported by the observation of a half field line, indicative for the presence of an $S \geq 1$ spin system. A comparison of the saturation behavior of the $\mathrm{N}$ acceptor in synthetic $\mathrm{MoS}_{2}$ with the As acceptor in natural $\mathrm{MoS}_{2}$ also revealed that the $\mathrm{N}$ signal saturates at a much lower incident power level, corresponding to $T_{1}(4.2 \mathrm{~K}) \approx$ $3 \times 10^{-4} \mathrm{~s}$. This different behavior can be attributed to the stronger spin-orbit coupling, and consequently better coupling to the crystal lattice, of the As center compared to the $\mathrm{N}$ center.

Assessing the stability of the $\mathrm{N}$ dopant, a variety of thermal treatments revealed drastic inactivation (passivation) of the $\mathrm{N}$ acceptor when exposed to an $\mathrm{H}_{2}$ ambient at $\sim 500^{\circ} \mathrm{C}$. Reactivation in vacuum of the $\mathrm{N}$ acceptor at temperatures up to $\sim 740{ }^{\circ} \mathrm{C}$ appeared unsuccessful, urging great caution with respect to doping efficiency when exposing $\mathrm{N}$-doped $\mathrm{MoS}_{2}$ to $\mathrm{H}_{2}$ at relatively high temperatures of $\gtrsim 500^{\circ} \mathrm{C}$.

Finally, device-electronically most relevant, extensive monitoring of the T-dependent ESR intensity at K- and Q-band revealed the $\mathrm{N}$ dopant as a shallow acceptor with an activation energy of $E_{a}=45 \pm 7 \mathrm{meV}$, uncovering $\mathrm{N}$ as an adequate candidate for stable covalently bonded p-type doping of bulk $\mathrm{MoS}_{2}$.

\section{ACKNOWLEDGMENTS}

This work received partial financial support by the ERA-NET 2Dfun project (2D functional $\mathrm{MX}_{2} /$ graphene heterostructures) within the framework of the EU Graphene Flagship.

${ }^{1}$ Q. H. Wang, K. Kalantar-Zadeh, A. Kis, J. N. Coleman, and M. S. Strano, Nat. Nanotechnol. 7, 699 (2012).

${ }^{2}$ D. Voiry, A. Mohite, and M. Chhowalla, Chem. Soc. Rev. 44, 2702 (2015).

${ }^{3}$ B. Radisavljevic, A. Radenovic, J. Brivio, V. Giacometti, and A. Kis, Nat. Nanotechnol. 6, 147 (2011).

${ }^{4}$ I. Song, C. Parkab, and H. C. Choi, RSC Adv. 5, 7495 (2015).

${ }^{5}$ S. McDonnell, R. Addou, C. L. Hinkle, and R. M. Wallace, in $2 D$ Materials for Nanoelectronics, edited by M. Houssa, A. Dimoulas, and A. Molle (Taylor \& Francis, Boca Raton, FL, 2016), p. 163.

${ }^{6}$ A. H. Castro Neto and K. Novoselov, Mater. Express 1, 10 (2011).

${ }^{7}$ J.-G. Song et al., Nat. Commun. 6, 7817 (2015).

${ }^{8}$ A. K. Geim and I. V. Grigorieva, Nature 499, 419 (2013).

${ }^{9}$ R. Ganatra and Q. Zhang, ACS Nano 8, 4074 (2014).

${ }^{10}$ X. Cui et al., Nat. Nano 10, 534 (2015).

${ }^{11}$ K. Dolui, I. Rungger, C. Das Pemmaraju, and S. Sanvito, Phys. Rev. B 88, 075420 (2013).

${ }^{12}$ Q. Yue, S. Chang, S. Qin, J. Li, Phys. Lett. A 377, 1362 (2013).

${ }^{13}$ H.-P. Komsa and A. V. Krasheninnikov, Phys. Rev. B 91, 125304 (2015).

${ }^{14}$ M. S. Wu, B. Xu, G. Liu, and C.-Y. Ouyang, Acta Phys. Sin. 62, 037103 (2013).

${ }^{15}$ H. Shu, P. Luo, P. Lang, D. Cao, and X. Chen, ACS Appl. Mater. Interfaces 7, 7534 (2015).

${ }^{16}$ D. Ma, W. Ju, T. Li, X. Zhang, C. He, B. Ma, Y. Tang, Z. Lu, and Z. Yang, Appl. Surf. Sci. 364, 181 (2016).

${ }^{17}$ Y. C. Cheng, Z. Y. Zhu, W. B. Mi, Z. B. Guo, and U. Schwingenschloegl, Phys. Rev. B 87, 100401R (2013).

${ }^{18}$ A. Ramasubramaniam and D. Naveh, Phys. Rev. B 87, 195201 (2013).

${ }^{19}$ A. W. Robertson et al., ACS Nano 10, 10227 (2016).

${ }^{20}$ A. Molle et al., Adv. Electron. Mater. 2, 1600091 (2016).

${ }^{21}$ A. Nipane, D. Karmakar, N. Kaushik, S. Karande, and S. Lodha, ACS Nano 10, 2128 (2016).

${ }^{22}$ O. Brontvein, R. Tenne, and A. Enyashin, Inorganics 2, 363 (2014).

${ }^{23}$ R. S. Title and M. W. Shafer, Phys. Rev. Lett. 28, 808 (1972).

${ }^{24}$ R. S. Title and M. W. Shafer, Phys. Rev. B 8, 615 (1973).

${ }^{25}$ M. R. Laskar et al., Appl. Phys. Lett. 104, 092104 (2014).

${ }^{26}$ J. Suh et al., Nano Lett. 14, 6976 (2014).

${ }^{27}$ E. D. Brandao, G. M. Ribeiro, P. H. Vaz, J. C. González, and K. Krambrock, J. Appl. Phys. 119, 235701 (2016).

${ }^{28}$ S. Iacovo, A. Stesmans, M. Houssa and V. V. Afanas'ev, J. Phys. Condens. Matter 29, 08LT01 (2017).

${ }^{29}$ C. Huang, Y. Jin, W. Wang, L. Tang, C. Song, and F. Xiu, J. Semiconductors 38, 033004 (2017).

${ }^{30} \mathrm{~K}$. Zhang et al., Nano Lett. 15, 6586 (2015). 
${ }^{31}$ A. Stesmans, S. Iacovo, and V. V. Afanas'ev, Appl. Phys. Lett. 109, 172104 (2016).

${ }^{32}$ A. Azcatl et al., Nano Lett. 16, 5437 (2016).

${ }^{33}$ L. Yang et al., Nano Lett. 14, 6275 (2014).

${ }^{34}$ S. McDonnell, R. Addou, C. Buie, R. M. Wallace, and C. L. Hinkle, ACS Nano 8, 2880 (2014).

${ }^{35}$ A. T. Neal, R. Pachter, and S. Mou, Appl. Phys. Lett. 110, 193103 (2017).

${ }^{36}$ B. Schoenaers, A. Stesmans, and V. V. Afanas'ev, AIP Adv. 7, 105006 (2017).

${ }^{37}$ B. Schoenaers, A. Stesmans, and V. V. Afanas'ev, Phys. Status Solidi C 14, 1700211 (2017).

${ }^{38}$ H. Ue and S. Maekawa, Phys. Rev. B3, 4232 (1971); J. D. Quirt and J. R. Marko, Phys. Rev. B7, 3842 (1973).

${ }^{39}$ C. P. Slichter, Phys. Rev. B 99, 479 (1955).

${ }^{40}$ HQ Graphene, Groningen, The Netherlands.

${ }^{41}$ A. Stesmans, Phys. Rev. B 48, 2418 (1993).

${ }^{42}$ J. I. Pankove, D. E. Carlson, J. E. Berkeyheiser, and R. O. Wance, Phys. Rev. Lett. 51, 2224 (1983).

${ }^{43}$ A. Stesmans and Y. Wu, J. Phys. D Appl. Phys. 21, 1205 (1988).
${ }^{44}$ A. Stesmans, J. Magn. Reson. 76, 14 (1988).

${ }^{45}$ J. A. Weil, J. R. Bolton, and J. E. Wertz, Electron Paramagnetic Resonance: Elementary Theory and Practical Applications (Wiley, NY, 1994), pp. 504-507.

${ }^{46}$ Trade name of Heraeus, Inc.

${ }^{47}$ A. Stesmans and F. Scheerlinck, Phys. Rev. B 51, 4987 (1994).

${ }^{48}$ T. G. Castner, Jr., Phys. Rev. 115, 1506 (1959).

${ }^{49}$ C. P. Poole, Jr., Electron Spin Resonance: A Comprehensive Treatise on Experimental Techniques (Dover, NY, 1983), pp. 589-600.

${ }^{50}$ A. Stesmans, Phys. Rev. B 61, 8393 (2000).

${ }^{51}$ J. S. Blakemore, Semiconductor Statistics (Pergamon, Oxford, 1962).

${ }^{52}$ E. Scalise, M. Houssa, G. Pourtois, V. V. Afanas'ev, and A. Stesmans, Physica E 56, 416 (2014); E. Scalise, in $2 D$ Materials for Nanoelectronics, edited by M. Houssa, A. Dimoulas, and A. Molle (Taylor \& Francis, London, 2016), p. 141.

${ }^{53}$ T. Cheiwchanchamnangij and W. Lambrecht, Phys. Rev. B 85, 205302 (2012).

${ }^{54}$ D. A. Neamen, Semiconductor Physics and Devices (Irwin, Boston, 1992). 